FORMATION Formation emploi

Revue française de sciences sociales

139 | Juillet-Septembre 2017

De l'autonomie dans les parcours professionnels

\title{
Trajectoires professionnelles et géographiques : l'étude de trois générations de docteurs
}

Career paths and geographical trajectories: the analysis of three PhD generations

Berufliche und geografische Laufbahn: Untersuchung an drei Ärztegenerationen Trayectorias profesionales y geográficas: el estudio de tres generaciones de doctores

\section{Bastien Bernela}

\section{OpenEdition}

Journals

Édition électronique

URL : http://journals.openedition.org/formationemploi/5154

DOI : 10.4000/formationemploi.5154

ISSN : 2107-0946

Éditeur

La Documentation française

Édition imprimée

Date de publication : 15 octobre 2017

Pagination : $147-170$

ISSN : 0759-6340

Référence électronique

Bastien Bernela, «Trajectoires professionnelles et géographiques : l'étude de trois générations de docteurs », Formation emploi [En ligne], 139 | Juillet-Septembre 2017, mis en ligne le 15 octobre 2019, consulté le 30 octobre 2020. URL : http://journals.openedition.org/formationemploi/5154 ; DOI : https://doi.org/10.4000/formationemploi.5154 


\title{
Trajectoires professionnelles et géographiques : l'étude de trois générations de docteurs
}

\author{
BASTIEN BERNELA \\ Maître de Conférences en sciences économiques, CRIEF (Centre de recherche sur \\ I'intégration économique et financière) EA2249, université de Poitiers
}

Résumé

Trajectoires professionnelles et géographiques : l'étude de trois générations de docteurs

L'accès à l'emploi scientifique des jeunes docteurs est une préoccupation récurrente dans les travaux empiriques récents. Si la question de la mobilité spatiale des chercheurs fait également débat, ce sont souvent deux problématiques indépendantes. Nous proposons une analyse conjointe des déterminants des trajectoires professionnelles et géographiques des docteurs à partir des données de trois enquêtes Génération (2001, 2004, 2007) du Céreq. Les statistiques descriptives et l'estimation d'un probit bivarié mettent en évidence un renforcement des mobilités géographiques des docteurs, ainsi que l'importance croissante des publications pour l'accès à l'emploi académique.

Mots-clés : doctorat ; trajectoire d'insertion ; mobilité géographique ; métier de la recherche; segmentation du marché du travail

Abstract

\footnotetext{
Career paths and geographical trajectories: the analysis of three PhD generations

Access of young PhDs to scientific position is a recurrent concern in recent empirical works. The issue of spatial mobility of researchers is also often discussed, but rarely both simultaneously. We propose a joint analysis of the determinants of occupational and geographical PhDs paths by using three Céreq Generation surveys (2001, 2004, 2007). Descriptive statistics and estimation of a bivariate probit highlight the strengthening of PhDs geographical mobility, and the growing importance of publications for access to academic employment.
}

Keywords: phd ; pathway to integration ; geographic mobility; occupation in research ; labour market segmentation

Journal of Economic Literature: J 24 ; J 62

Traduction : Auteur. 
Après une longue expérience diplômante de la recherche, les docteurs détiennent des connaissances scientifiques et techniques qu'il est primordial de diffuser entre les secteurs d'activité et dans l'espace. Dès lors, la question de la mobilité est centrale dans les débats liés à l'emploi des jeunes en début de carrière, qu'il s'agisse d'une mobilité professionnelle (changement de secteur d'activité) ou géographique (changement de localisation).

Du côté de la mobilité professionnelle, les trajectoires observées interrogent la capacité du système de recherche français à former des docteurs qui sauront s'insérer dans le public comme dans le privé. Si la carrière académique a longtemps été le débouché naturel du doctorat, de nombreux auteurs estiment que le changement de gouvernance de la science doit mener à une professionnalisation du doctorat et à de plus fortes collaborations avec le monde socio-économique (Gibbons et al., 1994 ; Etzkowitz et Leydesdorff, 2000).

Quant à la mobilité géographique des docteurs, elle est pour beaucoup un gage de circulation des connaissances (Almeida et Kogut, 1999 ; Autant-Bernard et Massard, 2001) : l'accès aux carrières académiques serait d'ailleurs de plus en plus soumis à une injonction à la mobilité (Vincent et al., 2010), révélatrice de la transformation de notre société de manière plus générale (Boltanski et Chiapello, 1999 ; Bauman, 2013).

Les trajectoires professionnelles et géographiques des chercheurs en début de carrière présentent donc toutes deux des enjeux certains, que les travaux empiriques ont pour habitude d'étudier séparément. Or, on voit bien qu'elles sont difficiles à démêler, comme l'illustre le débat des universitaires autour du recrutement local (Bouba-Olga et al., 2008 ; Godechot et Louvet, 2008), évoquant une insertion facilitée des docteurs dans leur université de soutenance.

Dès lors, il semble important de mieux comprendre les modalités d'accès aux différentes carrières possibles après le doctorat et dans quelle mesure celui-ci s'accompagne ou non d'une mobilité géographique. Nous proposons d'établir une distinction essentielle entre les docteurs qui s'insèrent dans l'enseignement supérieur et la recherche et les autres (dans la suite de l'article, nous parlerons de docteurs ESR versus docteurs non ESR), et d'identifier les déterminants d'accès à la carrière académique. Nous interrogerons simultanément l'impact de ces mêmes variables sur les comportements de mobilité géographique des jeunes docteurs. Nous proposons une analyse dynamique de ces trajectoires afin d'observer l'évolution de l'effet d'une même variable. Il s'agit d'observer si les déterminants de l'accès à l'ESR et de la mobilité géographique ont des effets stables dans le temps, s'ils s'estompent ou s'ils s'intensifient.

Empiriquement, cette recherche repose sur le traitement des enquêtes Génération du Céreq, et plus précisément des générations 2001, 2004 et 2007¹, pour lesquelles

1. À notre connaissance, seuls Béret et $a$ l. (2004) ont réalisé une analyse groupée de plusieurs enquêtes 
nous disposons d'un ensemble d'informations relatives au parcours de recherche des docteurs.

À l'aune de la littérature et des résultats empiriques existants, nous identifions les principaux déterminants des trajectoires professionnelles et géographiques des docteurs. Puis nous présentons les données et les variables mobilisées (sociodémographiques, géographiques et caractéristiques du doctorat) pour étudier les trajectoires des docteurs. À l'aide d'un modèle probit bivarié, nous introduisons les principaux résultats.

\section{De l'intérêt de croiser les trajectoires professionnelles et géographiques des docteurs}

Nous revenons ici sur les travaux qui s'intéressent, de manière distincte, aux déterminants de la trajectoire professionnelle des docteurs, d'une part, et de leur trajectoire géographique, d'autre part. Puis, nous justifions le positionnement original de cet article qui consiste à les étudier conjointement.

\subsection{Les docteurs sont confrontés à une forte segmentation des marchés du travail}

Conformément au système LMD (licence-master-doctorat) établi à l'échelle européenne, le doctorat dispense le plus haut niveau de diplôme. Si l'on considère que le diplôme protège de la précarité, les docteurs devraient constituer une catégorie de population privilégiée en termes d'accès à l'emploi et de qualité des emplois occupés. Or, les études des trajectoires professionnelles des jeunes docteurs mettent en évidence leur relative difficulté à accéder à la recherche privée et à se stabiliser sur le marché de l'emploi académique (Bonnal et Giret, 2009 ; Auriol, 2010).

On observe une tendance à la baisse du nombre de postes de maîtres de conférences mis au concours, alors que le nombre de qualifiés est stable. Ainsi, entre 2006 et 2011, le nombre de postes ouverts au recrutement est passé de 2300 à 1700 et le vivier des personnes qualifiées est resté stable, autour de 6450 (MESR, 2012 ; MESR, 2013). Malgré ce contexte de plus en plus concurrentiel, les carrières académiques présentent une attractivité certaine (Stephan, 1996 ; APEC, 2014). Face à la rareté des postes dans l'ESR dans de nombreuses disciplines, les étudiants pourraient être moins attirés par la carrière universitaire en raison de la difficulté attendue pour se stabiliser (Fox et Stephan, 2001), mais selon Roach et Sauermann (2010), c'est moins l'offre réelle de postes qui importe que la perception que les docteurs ont de ce marché du travail.

Génération du Céreq. 
De nombreux travaux (Mangematin, 2000 ; Giret et al., 2007 ; Bonnal et Giret, 2009) concluent à la forte segmentation du marché du travail des docteurs entre carrières académiques et carrières dans le privé, dont l'accès dépend de critères de recrutement différenciés. À partir d'une étude de cas de 400 jeunes docteurs en sciences de l'ingénieur à Grenoble, Mangematin (op. cit.) montre que les doctorants construisent leur parcours en fonction du type d'emploi qu'ils espèrent (recherche publique versus privée), et valorisent leurs résultats en fonction de leur projet professionnel. Ainsi, tous les doctorants ne se conforment pas aux critères de recrutement académique, mais à ceux du monde professionnel dans lequel ils anticipent de s'insérer en fin de thèse.

Les conditions de réalisation de la thèse vont fortement structurer la trajectoire. Parmi elles, le type de financement joue un rôle prédominant. Louvel (2006) montre ainsi la diversité des pratiques de recherche entre les allocataires localisés dans les laboratoires publics et les doctorants salariés des entreprises. D'ailleurs, selon Bonnal et Giret (op. cit.), "les thésards ayant bénéficié d'une allocation de recherche et d'un poste de moniteur, initialement sélectionnés sur des critères scolaires lors de leur entrée en doctorat, sont ceux qui accèdent le plus rapidement à l'emploi académique». Par ailleurs, Giret et al. (op. cit.) montrent que l'obtention d'une allocation de recherche n'a aucun effet sur la rémunération du jeune docteur dans le privé, le profil de doctorant qui bénéficie des meilleures conditions d'insertion dans ce secteur étant les CIFRE (Convention Industrielle de Formation par la Recherche). Bonnard (2012) montre également que le doctorat est un déterminant essentiel pour accéder à la recherche-développement privée, mais uniquement s'il s'accompagne d'une expérience en entreprise durant la thèse.

Bonnal et Giret (op. cit.) se sont intéressés aux facteurs expliquant la stabilisation des jeunes docteurs sur le marché de l'emploi académique français. À partir de l'enquête Génération 2001 du Céreq, ils mettent en évidence le rôle central des publications dans les mécanismes d'évaluation des chercheurs. "Le recrutement à partir des publications en début de carrière paraît d'autant plus pertinent que les inégalités de publications augmentent en cours de carrière au profit de ceux qui ont le plus publié au début ", une forte activité de publication pendant le doctorat signalerait donc une plus grande productivité future. Conformément à la forte segmentation du marché du travail des docteurs, l'activité de publication pendant la thèse n'influence pas l'accès à l'emploi dans le privé (Mangematin, op. cit.; Giret et al., op. cit.).

Un autre déterminant de l'accès des docteurs à l'emploi académique, largement étudié dans la littérature, est le post-doctorat. Passage obligé dans certaines disciplines, il génère une file d'attente entre les générations de docteurs (Robin et Cahuzac, 2003). Comme le précisent Bonnal et Giret (op. cit.), soutenir sa thèse entre février et août augmente fortement la probabilité de réaliser un post-doctorat, l'absence d'échéances professionnelles à court terme - étant donné le calendrier de qualification et de candidature aux postes de maître de conférences notamment - favorisant le départ en stage postdoctoral (Recotillet, 2007). 
Les trajectoires des docteurs sont également marquées par un effet de discipline. Pour les jeunes docteurs, la probabilité de trouver un emploi académique va fortement dépendre du rapport entre le nombre de postes ouverts au concours dans les sections concernées et le nombre de candidats qualifiés. Dans des disciplines comme la chimie ou les sciences de la vie et de la terre, où le marché du travail académique est tendu, le post-doctorat s'est développé comme un contrat transitoire. L'opportunité de pouvoir s'insérer hors ESR diffère également fortement d'une discipline à l'autre (Riedinger et Zaiem, 2011). Les docteurs sont parfois en concurrence directe avec les sortants des grandes écoles de niveau bac +5 (écoles d'ingénieurs en sciences et écoles de commerce en économie/gestion).

Enfin, Bonnal et Giret (op. cit.) montrent que «les jeunes docteurs ont une probabilité plus élevée d'accéder à l'emploi académique dans la région où ils ont obtenu leur doctorat, ce que l'on peut considérer comme un indice d'une préférence relative pour le recrutement local ", la trajectoire professionnelle faisant alors intervenir des déterminants géographiques.

\subsection{Trajectoires géographiques et comportement de mobilité}

La question de l'insertion professionnelle abordée dans la partie précédente laisse souvent peu de place à celle de la géographie des trajectoires. Traditionnellement, l'économie s'est intéressée aux mobilités dans le cadre des théories du job search. Ces dernières considèrent la mobilité comme un choix rationnel résultant d'une comparaison coûts/bénéfices. Il y a migration seulement si les individus obtiennent i) un emploi s'ils sont au chômage ou ii) une augmentation de salaire s'ils sont déjà en emploi (Sjaastad, 1962 ; Lippman and McCall, 1976), ce que Drapier et Jayet (2002), ainsi que Lemistre et Magrini (2010) confirment empiriquement à partir de données Céreq.

La théorie du capital humain (Becker, 1962) ajoute que plus un individu est qualifié et fortement doté en capital humain, plus la rentabilisation de l'investissement dans ce capital amène à un consentement pour la mobilité. Empiriquement, Arntz (2010) a mis en évidence que la variabilité des salaires est une fonction croissante du degré de qualification des emplois et du niveau de diplôme des individus, suggérant que la recherche d'emploi, pour les plus qualifiés, s'opère à une échelle spatiale plus large. Si l'on approxime l'investissement en capital humain par la durée d'études, les docteurs devraient donc être particulièrement mobiles. Dans le cas des carrières académiques, les mobilités pour raison salariale sont moins probables dans la mesure où les rémunérations sont encadrées à l'échelon national.

Si pour l'économie standard du travail, l'espace est simplement un paramètre permettant d'optimiser la recherche d'emploi, Herzog et al. (1993) l'intègrent différemment en introduisant la notion de coûts de prospection et de migration qui augmentent avec 
la distance. Plus précisément, Schwartz (1973) considère que les coûts de migration se composent de coûts psychologiques, comme l'éloignement avec la famille et les amis. Cet argument est repris par la théorie du capital social local (David et al., 2010) : "Si les individus se sentent fortement attachés à une ville ou une région, ils investiront dans $d u$ capital social local, parce que le rendement de ces relations locales est élevé (...) Un haut niveau de capital social local augmente le cồt de la mobilité et réduit en retour les incitations à migrer. Le capital social local joue toujours négativement sur la mobilité » (p. 3, traduit par nous). On peut penser que cet ancrage territorial est renforcé lorsque les docteurs réalisent leur thèse dans leur région d'origine, comme l'a montré Grossetti (1991) dans le cadre d'une étude de cas sur une population approchante d'ingénieurs toulousains. Selon Perret (2003), avoir soutenu dans sa région d'origine augmente significativement la probabilité d'y rester en emploi. Ainsi, près de quatre docteurs sur dix n'ont jamais changé de région entre la classe de $6^{\text {ème }}$ et le premier emploi après la thèse.

La situation matrimoniale constitue également un élément déterminant des trajectoires géographiques des individus, puisqu'elle fait peser sur les choix de localisation un ensemble de contraintes. En effet, plusieurs auteurs (Mincer, 1978 ; Shauman et Xie, 1996 ; Vincent et al., 2010 ; Boussard, 2013) ont montré que les personnes en couple sont soumises à des processus décisionnels joints qui compliquent la mobilité (emploi du conjoint, enfants scolarisés, etc.). Ces contraintes sont particulièrement fortes pour les docteurs, qui finissent leurs études à un âge avancé dans le cycle de vie 29 ans en moyenne -, âge auquel intervient souvent l'établissement de projets comme la mise en couple, la parentalité, un achat immobilier, etc., facteurs d'ancrage spatial et relationnel.

Au total, la littérature existante a fourni un ensemble de résultats qui ont permis de mieux comprendre l'accès des jeunes docteurs aux carrières académiques ainsi que leur comportement de mobilité. Ces deux phénomènes ont été traités empiriquement de façon indépendante, ne permettant pas de tester l'existence d'effets simultanés de certaines variables sur les trajectoires professionnelles et géographiques des docteurs. À partir d'un même jeu de données, nous déterminons dans quelle mesure i) les jeunes docteurs accèdent à l'ESR et ii) ces carrières académiques sont locales. Observe-t-on des déterminants (d'ordre sociodémographique ou liés à l'expérience de doctorat) qui influencent à la fois le type de carrière et la géographie de cette carrière ? L'analyse conduite sur trois générations permet également de tester la stabilité des déterminants dans le temps. Nous présentons, dans la partie suivante, les données mobilisées. 


\section{Une analyse dynamique des déterminants des trajectoires à l'aune de trois générations de docteurs}

Les données mobilisées proviennent de l'appariement de trois enquêtes du Céreq sur les générations 2001, 2004 et 2007 (cf. encadré 1). Elles rassemblent ainsi plus de 72000 individus interrogés trois ans après leur sortie du système éducatif français, respectivement en 2004, 2007 et 2010. Nous nous centrons dans cet article sur les docteurs en emploi au moment de l'interrogation, c'est-à-dire trois ans après la fin de leur thèse, soit près de 30000 docteurs après pondération (cf. tableau 1).

\section{Encadré 1 : L'appariement de trois enquêtes Génération du Céreq}

Le choix de ces trois enquêtes Génération (G2001/G2004/G2007) repose sur le fait qu'elles présentent, contrairement aux précédentes enquêtes, un ensemble d'informations relatives au parcours de recherche des docteurs : la discipline de leur thèse, l'obtention ou non d'un financement durant la thèse, le nombre de publications dans des revues à comité de lecture à l'issue de la thèse, leur projet professionnel en fin de thèse, et la réalisation ou non d'un post-doctorat. Le tableau 1 détaille les effectifs représentés dans chacune des enquêtes : les poids de chaque génération sont quasi-équivalents, n'introduisant pas de surreprésentation d'une génération vis-à-vis des autres. Le Céreq utilise une pondération afin que les répondants soient représentatifs de la population sortante du système éducatif.

Tableau 1 : Taille de la population étudiée par génération

\begin{tabular}{l|cc|cc}
\hline & \multicolumn{2}{|c|}{ Effectifs répondants } & \multicolumn{2}{c}{ Effectifs pondérés } \\
\hline G2001 & 1384 & $34 \%$ & 9313 & $32 \%$ \\
$\mathrm{G} 2004$ & 1506 & $38 \%$ & 8775 & $31 \%$ \\
$\mathrm{G} 2007$ & 1126 & $28 \%$ & 10747 & $37 \%$ \\
\hline Total & 4016 & $100 \%$ & 28835 & $100 \%$ \\
\hline
\end{tabular}

Lecture : Les 1506 docteurs diplômés en 2004 ayant répondu à l'enquête du Céreq sont représentatifs de 8775 docteurs de leur génération. Source : Enquêtes Génération du Céreq, 2001, 2004 et 2007.

\subsection{Variables sociodémographiques et géographiques}

Les variables individuelles d'ordre sociodémographique correspondent à l'âge, au genre, à l'origine sociale, au pays de naissance ${ }^{2}$ et à la situation matrimoniale des docteurs; elles sont présentées dans le tableau 2.

2. Les données indiquent le pays de naissance des individus et non leur nationalité. 
Tableau 2 : Statistiques descriptives des variables sociodémographiques

\begin{tabular}{l|ccc|c}
\hline \multicolumn{1}{c|}{ (en \%) } & G2001 & G2004 & G2007 & Total \\
\hline Age moyen (en années) & 29,2 & 28,8 & 29,0 & 29,0 \\
Homme & 51 & 59 & 53 & 54 \\
Père cadre & 47 & 49 & 51 & 49 \\
Né à l'étranger & 9 & 4 & 18 & 11 \\
En couple & 68 & 69 & 68 & 68 \\
Mobilité entre 6ème et doctorat & 49 & 50 & 55 & 51 \\
Ile-de-France & 32 & 32 & 36 & 34 \\
\hline
\end{tabular}

Lecture : $59,0 \%$ des docteurs diplômés en 2004 sont des hommes.

Source : Enquêtes Génération du Céreq, 2001, 2004 et 2007.

La population obtient le grade de docteur à l'âge de 29 ans en moyenne, âge qui n'est pas significativement différent entre hommes et femmes. Si la répartition en termes de genre est relativement équilibrée sur le total des docteurs enquêtés, les femmes sont largement surreprésentées en lettres et sciences humaines (62\%) et sous-représentées en sciences (37\%). Concernant l'origine sociale des docteurs, près de la moitié d'entre eux ont un père cadre (ou de profession intermédiaire), alors que cette proportion est seulement de $31 \%$ pour l'ensemble des sortants du système éducatif des trois générations étudiées : l'accès au doctorat est donc plus fréquent pour des individus originaires de familles aux catégories socioprofessionnelles supérieures. Par ailleurs, $10 \%$ environ des docteurs sont nés à l'étranger, proportion relativement instable entre les générations, passant de $4 \%$ pour la génération 2004 à $18 \%$ pour la génération 2007. Enfin, plus des deux-tiers des docteurs sont en couple au moment de l'enquête, proportion stable entre les générations.

Enfin, deux variables fournissent des indications sur la géographie des trajectoires des docteurs. Environ un docteur sur deux réalise sa thèse dans la région où il était localisé en classe de sixième, ce qui peut contribuer à l'acquisition d'un capital social local. Un docteur sur trois soutient sa thèse en Ile-de-France, confirmant la forte concentration des activités de recherche dans la région capitale.

\subsection{Variables liées au doctorat}

Contrairement aux autres diplômes, le doctorat est une expérience professionnelle en soi, de longue durée, dont le déroulement va fortement influencer la trajectoire post-doctorat. Le tableau 3 présente un ensemble de variables - par groupe disciplinaire ${ }^{3}$ - permettant

3. Concernant la répartition des thèses par discipline, nous avons fait le choix de retenir la catégorisation du Conseil National des Universités en trois grands secteurs disciplinaires, comme l'ont fait Bonnal et Giret (2009). Sur les trois générations étudiées, $17 \%$ des thèses relèvent du secteur "Droit, économie, gestion " (DEG), 24 \% du secteur « Lettres, sciences humaines » (LSH) et 59 \% du secteur « Sciences ». 
de caractériser la nature du doctorat, et dont l'impact sur les trajectoires professionnelles et géographiques des docteurs sera testé dans la partie suivante.

Tableau 3 : Statistiques descriptives des variables liées au doctorat, par secteur disciplinaire

\begin{tabular}{|c|c|c|c|c|}
\hline (en \%) & $\begin{array}{c}\text { Droit, } \\
\text { économie, } \\
\text { gestion }\end{array}$ & $\begin{array}{c}\text { Lettres, } \\
\text { sciences } \\
\text { humaines }\end{array}$ & Sciences & Total \\
\hline \multicolumn{5}{|l|}{ Durée de la thèse } \\
\hline 3 ans & 12 & 12 & 39 & 28 \\
\hline 4 ans & 23 & 27 & 45 & 37 \\
\hline 5 ans et plus & 65 & 61 & 16 & 35 \\
\hline \multicolumn{5}{|l|}{ Financement } \\
\hline Bourse avec enseignement & 61 & 46 & 35 & 42 \\
\hline Bourse sans enseignement & 19 & 24 & 46 & 36 \\
\hline $\begin{array}{l}\text { CIFRE (Convention Industrielle de Formation par } \\
\text { la Recherche) }\end{array}$ & 8 & 6 & 16 & 12 \\
\hline Sans financement & 12 & 24 & 3 & 10 \\
\hline \multicolumn{5}{|l|}{ Nombre de publications } \\
\hline 0 & 53 & 36 & 22 & 31 \\
\hline 1 & 13 & 17 & 18 & 17 \\
\hline 2 & 14 & 16 & 20 & 18 \\
\hline 3 & 11 & 13 & 15 & 14 \\
\hline 4 & 3 & 6 & 8 & 6 \\
\hline 5 et plus & 6 & 12 & 17 & 14 \\
\hline \multicolumn{5}{|l|}{ Projet professionnel } \\
\hline Enseignement supérieur et recherche & 81 & 86 & 65 & 73 \\
\hline Recherche privée & 5 & 2 & 19 & 13 \\
\hline Autre projet & 14 & 12 & 16 & 14 \\
\hline \multicolumn{5}{|l|}{ Post-doctorat } \\
\hline Oui & 13 & 12 & 47 & 33 \\
\hline Non & 87 & 88 & 53 & 67 \\
\hline
\end{tabular}

Lecture : $24 \%$ des docteurs en lettres et sciences humaines ont réalisé leur thèse non financée.

Source : Enquêtes Génération du Céreq, 2001, 2004 et 2007.

Concernant la durée de réalisation du doctorat, seulement $28 \%$ des thèses sont soutenues en trois ans. Elle varie fortement en fonction des secteurs disciplinaires : $39 \%$ des thèses en sciences sont soutenues en trois ans, contre seulement $12 \%$ en DEG et LSH.

La question du financement est essentielle parce qu'elle informe sur le degré d'immersion du doctorant dans un monde professionnel (thèse financée ou non, puis recherche académique en laboratoire versus recherche privée en entreprise). Ainsi, près d'une thèse sur dix n'est pas financée, là encore avec de fortes différences disciplinaires (3\% en sciences, $12 \%$ en DEG et $24 \%$ en LSH). Nous avons également introduit dans cette variable la question 
de l'enseignement : on peut considérer que l'exercice ou non d'une activité d'enseignement durant la thèse est un déterminant essentiel d'accès à l'emploi académique.

Le nombre de publications parues à la fin de la thèse met également en évidence l'hétérogénéité du profil des doctorants. Sept docteurs sur dix publient au moins un article pendant leur thèse, proportion qui n’a fait qu'augmenter entre les trois générations, ce qui témoigne du rôle croissant de la publication dans les processus d'évaluation de la recherche. Les docteurs en sciences sont ceux qui publient le plus, devant ceux de LSH, puis de DEG.

L'enquête renseigne également sur le projet professionnel des docteurs en fin de thèse ${ }^{4}$ : Enseignement Supérieur et Recherche (73\%), recherche privée (13\%), et hors recherche $(14 \%)$. La stabilité de cette distribution entre les générations témoigne d'une appétence toujours aussi forte des docteurs pour les carrières académiques. Les docteurs en sciences sont ceux qui projettent le plus de travailler dans la recherche privée ou hors recherche (19\% et $16 \%$ ) alors que les docteurs en DEG et LSH souhaitent faire carrière dans l'ESR à plus de $80 \%$.

Enfin, nous avons vu précédemment que le post-doctorat pouvait impacter la qualité de l'insertion, et qu'il est devenu un passage obligé dans certaines disciplines pour accéder à des postes titulaires dans l'ESR. Environ un tiers des docteurs déclare avoir réalisé un post-doctorat, cette proportion atteignant presque $50 \%$ pour les docteurs en sciences. On observe une forte progression des contrats postdoctoraux au fil des trois générations, qui concerne toutes les disciplines.

\subsection{Variables à expliquer : accès à l'ESR et mobilité géographique}

Nous nous intéressons, dans cet article, aux trajectoires professionnelles et géographiques de jeunes docteurs et à leurs déterminants. Pour cela, nous avons construit deux variables binaires.

\section{- ESR}

La variable ESR prend la valeur 1 lorsque le docteur occupe un emploi dans le secteur de l'Enseignement Supérieur et de la Recherche, 0 sinon. Nous considérons que le secteur de l'ESR regroupe l'ensemble des emplois caractérisés par une activité d'enseignement et/ou de recherche dans une Université ou dans un laboratoire public, indépendamment du fait que cet emploi soit ou non titulaire (maitre de conférences, chargé de recherche, ingénieur de recherche, ingénieur d'études, assistant ingénieur, ATER-Attaché Temporaire d'Enseignement et de Recherche -, post-doctorant, etc.).

Giret (2005) propose une typologie en trois secteurs d'activité : les chercheurs dans le public, les autres professions du public, les emplois privés. Ici, nous avons fait le choix d'une variable binaire, les professions du public hors ESR (notamment les enseignants du

4. Il s’agit d'une variable déclarative que nous avons recodée. 
primaire et secondaire) sont regroupées avec les emplois du secteur privé. Les modalités pour lesquelles $E S R=O$ présentent donc une forte hétérogénéité que nous justifions par l'objectif d'identifier les déterminants d'accès aux carrières académiques versus les autres carrières.

Méthodologiquement, nous nous focalisons sur la situation des docteurs en emploi trois ans après la soutenance, sans tenir compte du premier emploi, ni du type d'emploi occupé. "L'emploi occupé immédiatement après la soutenance doit souvent être considéré comme transitoire pour les jeunes docteurs (...). La situation au moment de l'enquête, trois ans après l'obtention de la thèse, permet davantage de caractériser les situations à un moment où les trajectoires se stabilisent. " (Béret et al., op. cit.). Certes, cela ne permet pas de se prononcer sur la qualité de l'insertion, mais l'objectif de cet article est de se focaliser sur le domaine professionnel dans lequel les docteurs s'insèrent.

\section{- Mobilité}

La variable Mobilité prend la valeur 1 lorsque le docteur travaille dans une région différente de celle où il a soutenu sa thèse trois ans auparavant, 0 sinon. Le découpage académique $\mathrm{du}$ territoire français correspond au découpage régional, hormis trois cas : la région Ilede-France est divisée en trois académies (Créteil, Paris et Versailles), la région ProvenceAlpes-Côte-d'Azur en deux académies (Marseille et Nice) et la région Rhône-Alpes en deux académies (Grenoble et Lyon). Pour ces deux dernières régions, l'échelle de l'académie nous paraît plus adaptée dans la mesure où il existe deux villes universitaires éloignées au sein de la même région, l'étude des mobilités entre académies ne doit pas être occultée. À l'inverse, la région Ile-de-France est caractérisée par une forte concentration d'établissements dans un même continuum géographique : migrer d'une académie à l'autre n'implique pas de changement d'agglomération et ne constitue pas une mobilité spatialement significative. Autrement dit, dans la suite de l'article, le terme de région réfère à la nomenclature des régions administratives, à l'exception de Rhône-Alpes et Provence-Alpes-Côte-d'Azur, pour lesquelles nous retenons l'échelle académique.

Notons que cette étude se focalise sur les mobilités infranationales puisque les données ne permettent pas de capter les mobilités internationales. Il s'agit d'une limite empirique importante, bien que plusieurs recherches aient démontré que la mobilité internationale des chercheurs français est relativement faible (Guellec et Cervantes, 2002 ; Harfi et Mathieu, 2006).

Le tableau 4 croise les trajectoires professionnelles et géographiques des jeunes docteurs enquêtés. Ainsi, seulement un tiers des docteurs en activité travaillent trois ans après la fin de leur thèse dans une autre région que celle où ils ont soutenu, chiffre en phase avec des résultats d'études similaires sur d'autres données (Perret, op. cit.; Bernela et al., 2017). Quant à la trajectoire professionnelle des docteurs, $42 \%$ sont en poste dans l'Enseignement Supérieur et la Recherche. Le taux de mobilité des ESR est supérieur de quatre points à celui des non ESR (38 \% versus $34 \%)$. 
Tableau 4 : Croisement des variables Mobilité et ESR

\begin{tabular}{l|cc|c}
\hline \multicolumn{1}{c|}{ (en \%) } & ESR & Non ESR & Total \\
\hline Mobile & 16 & 20 & 36 \\
Non mobile & 26 & 38 & 64 \\
\hline Total & 42 & 58 & 100 \\
\hline
\end{tabular}

Lecture : $26 \%$ des docteurs des générations 2001, 2004 et 2007 sont - trois ans après leur soutenance - en emploi dans l'Enseignement supérieur et la Recherche et dans la région de leur soutenance.

Source : Enquêtes Génération du Céreq, 2001, 2004 et 2007.

Lorsque l'on étudie la mobilité géographique d'une population donnée, on peut s'interroger sur la spécificité de son comportement. Afin de comparer le niveau de mobilité des docteurs avec celui d'autres catégories de population, le graphique 1 présente les taux de mobilité par niveau de diplôme, pour les trois générations étudiées. Les diplômés de Master sont la catégorie la plus mobile, ce qui vient infirmer l'existence d'une relation positive linéaire entre mobilité et niveau de diplôme. En moyenne, le comportement de mobilité des docteurs est même plus proche de celui des diplômés de Licence que de Master.

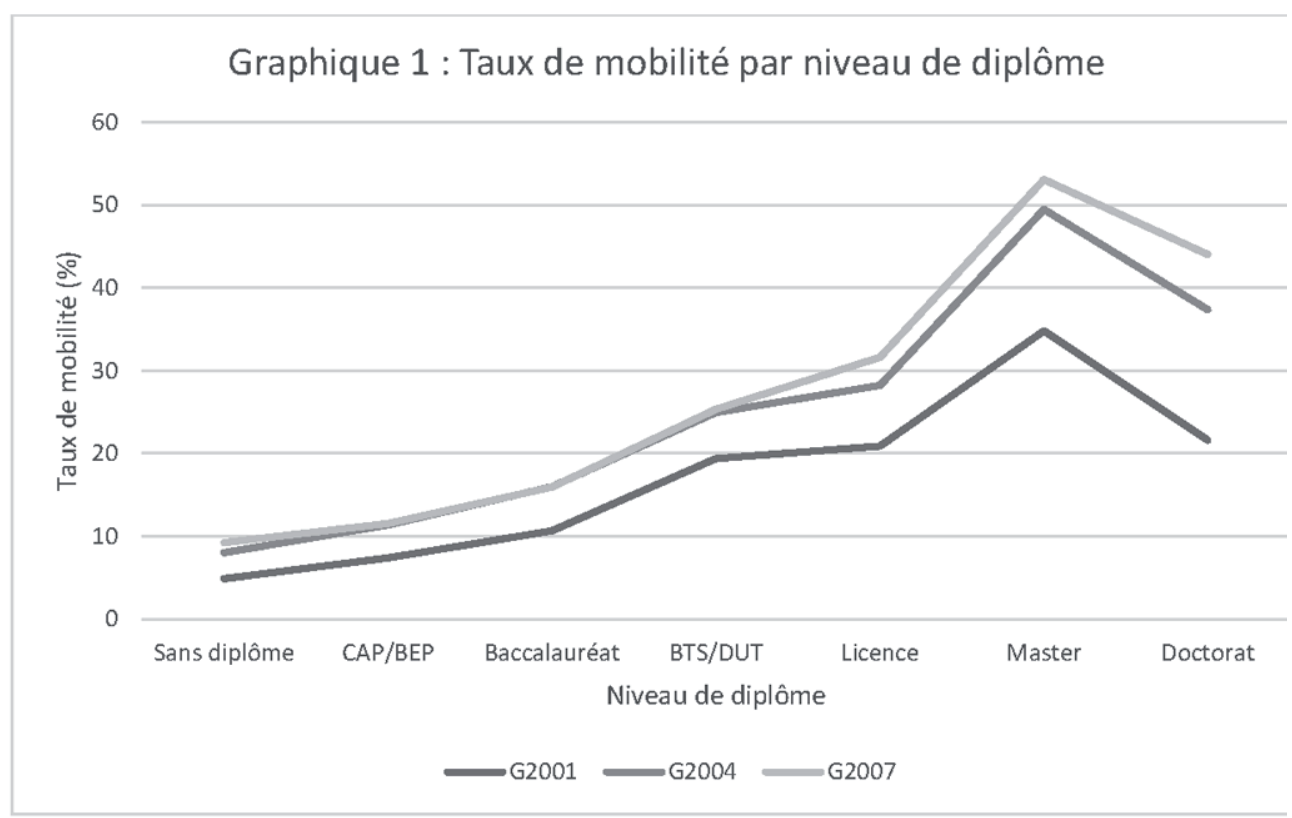

Lecture : $25 \%$ des BTS/DUT diplômés en 2004 sont en emploi dans une autre région que celle où ils ont terminé leurs études.

Source : Enquêtes Génération du Céreq, 2001, 2004 et 2007. 


\section{Accès à l'emploi académique et mobilité géographique : des déterminants différenciés}

Afin d'étudier conjointement les déterminants des trajectoires professionnelles et géographiques des jeunes docteurs, nous avons choisi d'estimer un probit bivarié (cf. encadré $\mathbf{2}$ ), dont les résultats sont présentés dans le tableau $\mathbf{5}$.

\section{Encadré 2 : Le choix du probit bivarié}

Comme on l'a vu, on ne peut pas déterminer a priori le sens de la causalité entre choix professionnel et choix de localisation, chaque individu pouvant être confronté à des opportunités et à des contraintes différentes. Econométriquement, cela signifie que chacune des deux variables ne peut pas être explicative de l'autre variable. Pour prendre en compte la simultanéité des événements au cours de la période et les biais éventuels d'endogénéité, nous avons recours à l'estimation d'un modèle probit bivarié (Lollivier, 2001 ; Debrand et Taffin, 2005). Chaque événement, en l'occurrence la trajectoire géographique et la trajectoire professionnelle du jeune docteur, est caractérisé par une variable binaire (cf. partie 2.3). Le modèle testé fait apparaître un coefficient de corrélation (rho), entre les résidus des deux équations probit, significativement différent (au seuil de $1 \%$ ). Cela justifie l'estimation d'un probit bivarié, plutôt que deux probit séparés. Le rho est positif, les variables inobservées jouent donc dans le même sens sur les deux variables à expliquer.

\subsection{La prise en compte des effets de génération}

Nous commentons d'abord l'effet de génération sur le modèle général. Alors que Béret et al. (op. cit.) avaient montré, en comparant plusieurs enquêtes Génération du Céreq, que l'accès des docteurs à la recherche publique ou privée n'est pas stable dans le temps (tendance à la baisse de la part des docteurs occupant un emploi d'enseignant-chercheur ou de chercheur dans le public), notre modèle n'indique aucune significativité des générations sur la probabilité d'accéder à l'ESR. Nos résultats soulignent une stabilisation de la part des carrières dans l'ESR, qui représente environ $42 \%$ pour chacune des trois générations étudiées 5 .

En revanche, on observe un net effet de génération sur la probabilité d'être mobile. Ainsi, le niveau de mobilité, c'est-à-dire la probabilité d'être en emploi trois ans plus tard dans une région différente de celle de soutenance, augmente significativement

5. Rappelons que nous nous focalisons sur les docteurs en activité, quelle que soit la nature de l'emploi occupé. La part des contrats stables (CDI - contrat à durée indéterminée - et fonctionnaires) des non-ESR (69 \%) est supérieure à celle des ESR (58\%) : la stabilisation semble donc plus difficile sur le marché de l'emploi académique, et les contrats précaires y sont plus nombreux (post-doctorat, ATER, etc.). 
dans le temps. Cette augmentation est très sensible, puisque le taux de mobilité passe de $23 \%$ en 2004, à $39 \%$ en 2007 et $45 \%$ en 2010, mouvement commun et d'amplitude égale pour les ESR et les non-ESR, le taux de mobilité des ESR restant supérieur de cinq points à celui des non ESR pour chacune des trois générations ${ }^{6}$.

Pour explorer les effets de génération, nous proposons de décliner les modèles bivariés par génération afin de retracer l'évolution de l'effet d'une même variable : l'objectif est de voir si les déterminants de l'accès à l'ESR et de la mobilité géographique ont des effets stables dans le temps, s'ils s'estompent ou s'ils s'intensifient. Notons que la distribution régionale des docteurs d'une génération à l'autre est stable et que les évolutions observées ne dépendent donc pas d'effets de structure géographique.

Une stratégie similaire nous amène à décliner le modèle en fonction des secteurs disciplinaires (docteurs en sciences humaines et sociales versus en sciences) puisque l'on peut s'attendre à des effets différenciés de certaines variables en fonction des disciplines. Dans la suite de la partie, nous proposons de discuter les résultats par groupe de variables, en apportant des éclairages complémentaires.

Tableau 5 : Résultats des estimations des probit bivariés

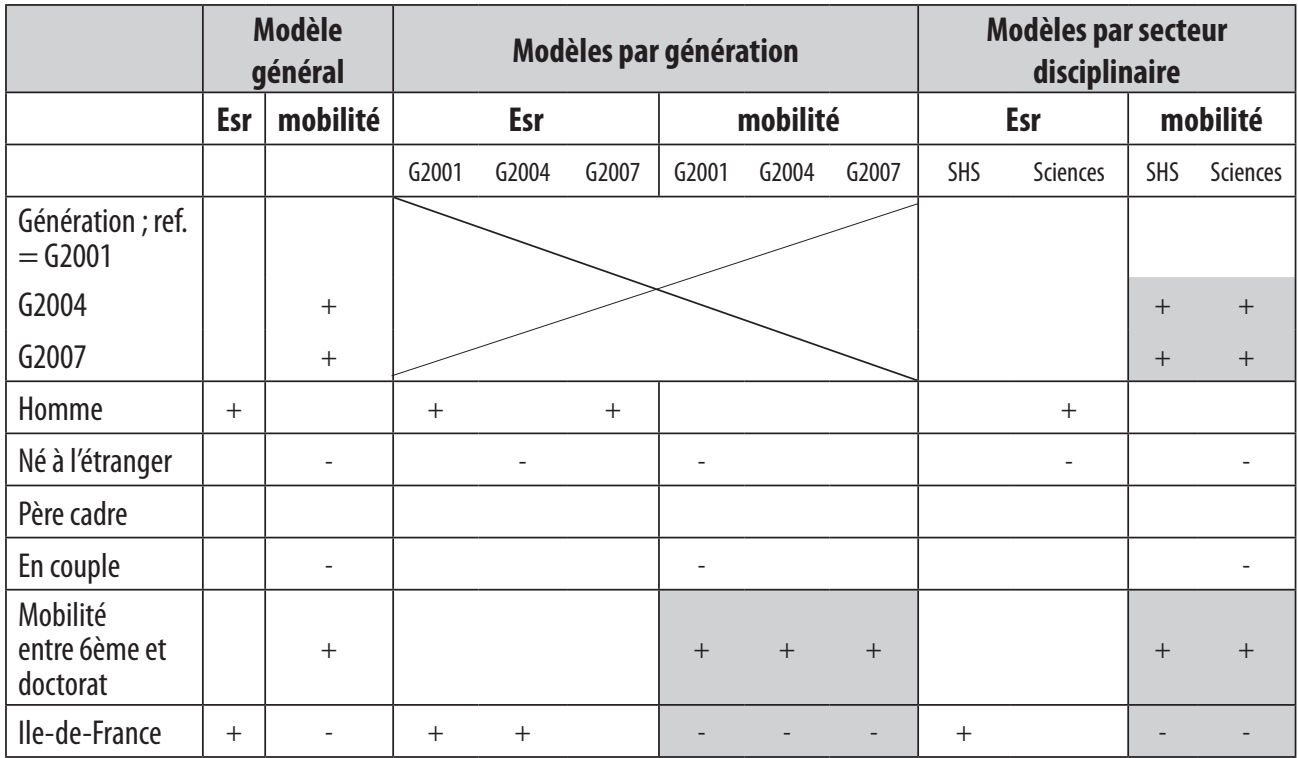

6. Comme on peut le constater sur le graphique 1, cette hausse de la mobilité touche de manière équivalente l'ensemble des niveaux de diplôme. 


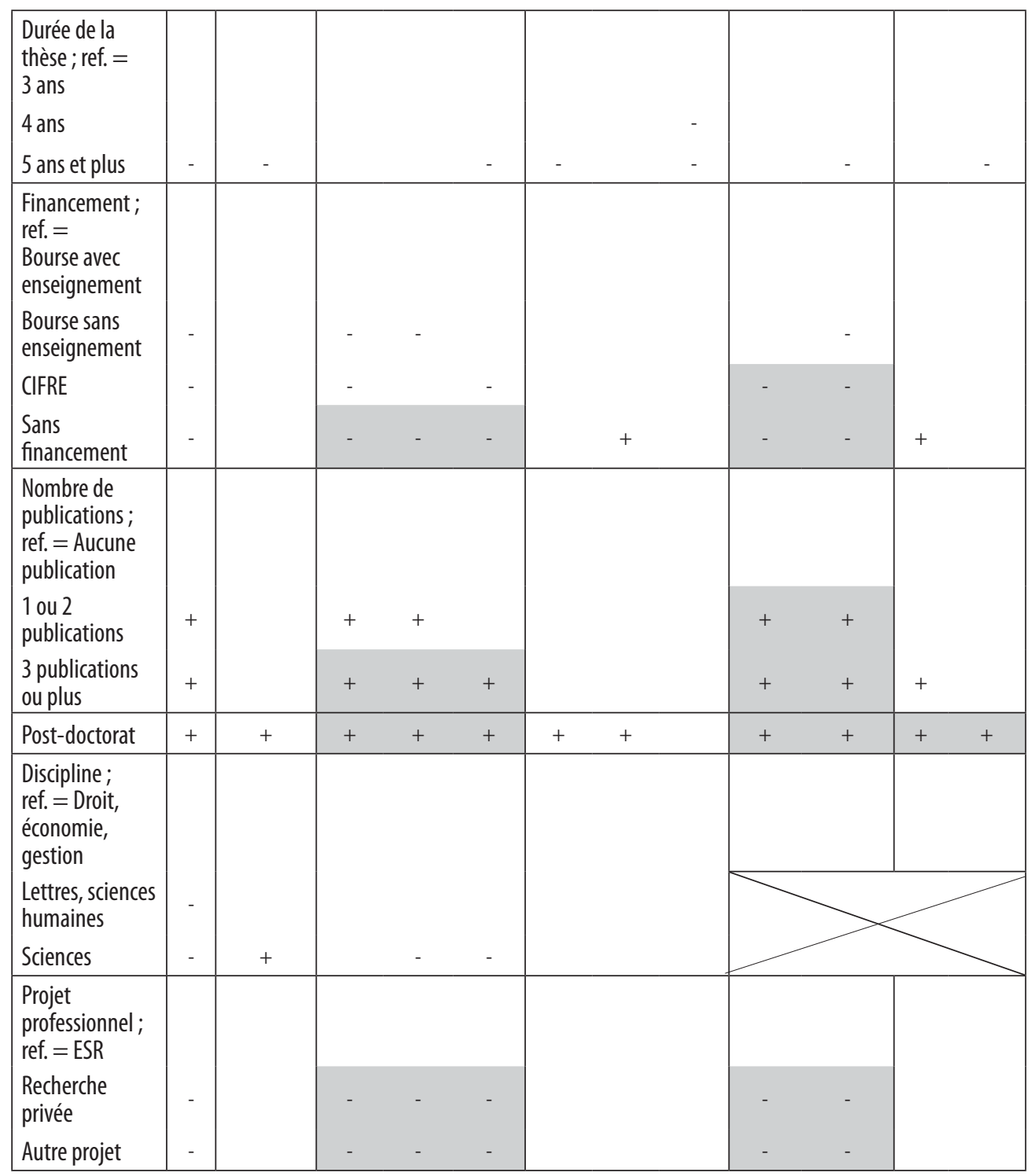

Note : Un signe (+/-) apparaît en cas de significativité (établie au seuil de 5 \%). Les zones surlignées en gris indiquent une stabilité des effets d'une même variable pour chaque génération ou chaque secteur disciplinaire.

Lecture : Avoir réalisé un post-doctorat augmente significativement la probabilité d'être en emploi trois ans après la soutenance dans l'ESR. Cet effet est observé pour les trois générations et pour les deux secteurs disciplinaires.

Source : Enquêtes Génération du Céreq, 2001, 2004 et 2007. 


\subsection{Le poids des variables sociodémographiques et géographiques révèle le degré d'ancrage des docteurs}

Tout d'abord, être un homme augmente fortement la probabilité d'accéder à l'ESR. Ce résultat confirme la plus grande difficulté, pour les femmes, à accéder au marché du travail académique, et laisse présager le plafond de verre observé lors de l'évolution de carrière (Marry, 2008). Cet effet est plus marqué en sciences, où la proportion d'hommes parmi les maîtres de conférences est sensiblement plus élevée (MESR, op. cit.). En revanche, le genre n'a pas d'effet sur la mobilité, alors que de nombreux travaux (Pochic, 2005 ; Bonnet et al., 2006) mettent en évidence que les femmes sont souvent moins mobiles que les hommes pour des raisons familiales. On peut supposer que pour les titulaires d'un doctorat, ces effets de genre sur le comportement de mobilité disparaissent.

En revanche, le fait d'être en couple a un effet négatif et significatif sur la mobilité, ce qui rejoint les conclusions de Béduwé (1994). Ce résultat met en évidence l'importance des déterminants d'ordre personnel ${ }^{7}$ sur la trajectoire professionnelle des individus (Grossetti, 1991 ; Dahl et Sorenson, 2010 ; Vincent et al., op. cit.). Être en couple n'influence par ailleurs pas la probabilité d'obtenir un poste dans l'ESR.

Être enfant de cadre n'a d'effet ni sur la mobilité, ni sur l'accès aux postes de l'ESR. Le capital culturel et social lié à l'origine des docteurs jouerait en amont de la thèse - la proportion d'enfants de cadres étant plus importante chez les docteurs relativement au reste de la population -, mais pas une fois le doctorat obtenu.

Les docteurs d'Ile-de-France ont une probabilité significativement plus élevée d'obtenir un poste académique et de rester dans leur région de soutenance. Pour la mobilité, ce résultat est particulièrement marqué puisqu'il concerne les trois générations et n'est pas sensible au secteur disciplinaire. Bernela et al. (op. cit.) ont montré qu'une part non négligeable des flux de docteurs, en France, concerne des mobilités infrafranciliennes, témoignant de l'existence d'un marché local du travail académique dans la région capitale. Dans un contexte de méfiance vis-à-vis du recrutement local, pour les jeunes docteurs, les opportunités de trouver un poste localement sans être recruté dans son université sont plus fortes dans des régions denses et à la structure d'emploi favorable. En effet, Drapier et Jayet (op. cit.) montrent que " chez les non-formés, le fait d'être originaire d'Ile-de-France incite significativement à la migration alors que l'on obtient un résultat opposé et tout aussi significatif chez les jeunes formés".

7. La question des enfants est importante lorsque l'on étudie la mobilité, mais cette variable est absente dans l'enquête génération 2007, c'est pourquoi elle n'est pas introduite dans l'analyse. En revanche, nous avons testé le modèle séparément pour les générations 2001 et 2004, en introduisant cette variable, et elle diminue significativement la probabilité d'être mobile, en plus du fait d'être en couple. 
On voit bien ici l'intérêt d'analyser conjointement les trajectoires professionnelles et géographiques des docteurs, puisqu'une même variable favorise la combinaison d'une carrière académique et locale : les opportunités plus importantes dans la région de soutenance du docteur permettent d'obtenir un emploi académique sans migrer. Notons que l'avantage relatif d'avoir réalisé son doctorat en Ile-de-France pour accéder à l'ESR disparaît pour la génération 2007. Ce résultat peut s'expliquer en partie par une diminution du nombre de postes académiques disponibles dans la région capitale, dans un contexte de déconcentration de la recherche (Grossetti et al., 2014).

Le fait d'avoir été mobile avant l'entrée en thèse - le docteur soutient dans une région différente de celle où il était localisé en classe de sixième ${ }^{8}$ - augmente très fortement la probabilité d'être mobile après la thèse', comme l'ont montré Baron et Perret (2008). L'hypothèse d'une compétence ou d'un apprentissage de la mobilité peut justifier ce résultat, dès lors que l'on considère que le fait d'avoir connu une première mobilité facilite l'expérience d'une seconde mobilité. Or, en examinant empiriquement les régions d'origine des docteurs et de destination après la thèse, on s'aperçoit qu'une part importante $(17 \%)$ des mobilités observées correspond à des retours. Une part non négligeable des mobilités ne concerne donc pas des mobilités «brutes » qui consisteraient à aller d'une région à une autre par hasard, mais un retour dans la région où le docteur a grandi. Dès lors, on peut calculer un taux de mobilité net en retranchant les retours du total des mobilités. Le tableau 6 révèle le poids des retours, pour chaque génération, et en distinguant les docteurs ESR et non ESR ; cela permet de dégager les taux de mobilités brut et net. On observe que les retours sont sensiblement plus fréquents chez les docteurs non ESR, ce qui contribue à creuser l'écart avec les docteurs ESR quand on examine le taux de mobilité net. De plus, les retours tendent à se renforcer dans le temps, expliquant partiellement la hausse du taux de mobilité brut décrite précédemment. Si l'on exclut les retours des cas de mobilité dans notre modèle (la variable à expliquer serait alors la mobilité nette), la variable de mobilité antérieure n'est plus significative, invalidant l'hypothèse d'un apprentissage de la mobilité.

Au final, le poids des retours atténue l'ampleur des mobilités, déjà faibles, et souligne un paradoxe statistique, puisque cette double mobilité est finalement le signe d'un ancrage. Ce résultat plaide pour une vision non binaire de la mobilité, afin de mieux prendre en compte la complexité de la trajectoire géographique d'un individu.

8. Les docteurs figurent parmi les jeunes sortants de l'Université qui ont le plus fréquemment changé de région durant leurs études (Perret, op. cit.).

9. En creux, cela signifie que faire sa thèse dans sa région d'origine renforce la probabilité d'y être en emploi trois années après la thèse, ce qui peut être le résultat d'un investissement dans le capital social local. 
Tableau 6 : Mobilité géographique et retour dans la région d'origine

\begin{tabular}{l|c|ccc|c}
\hline & (en \%) & G2001 & G2004 & G2007 & Total \\
\hline \multirow{3}{*}{ Taux de mobilité brut } & ESR & 25,8 & 40,7 & 48,1 & 38,8 \\
& Non ESR & 20,4 & 36,7 & 43,2 & 33,8 \\
& Total & 22,7 & 38,6 & 45,3 & 35,9 \\
\hline \multirow{2}{*}{ Part des retours dans les } & ESR & 8,7 & 17,4 & 11,4 & 12,8 \\
mobilités & Non ESR & 16,5 & 21,9 & 22,9 & 21,3 \\
& Total & 12,8 & 19,8 & 17,8 & 17,4 \\
\hline \multirow{3}{*}{ Taux de mobilité net } & ESR & 23,4 & 33,6 & 42,6 & 33,8 \\
& Non ESR & 17 & 28,6 & 33,3 & 26,6 \\
& Total & 19,8 & 31,0 & 37,3 & 29,6 \\
\hline
\end{tabular}

Lecture : Parmi les docteurs de la génération 2004, 38,6\% sont en emploi dans une autre région que celle où ils ont soutenu leur thèse. Parmi ces docteurs mobiles, 19,8\% sont retournés dans leur région d'origine, qu'ils avaient quittée pour leur thèse. Autrement dit, 31 \% des docteurs de la génération 2004 sont en emploi dans une région différente de leur région d'origine et de leur région de soutenance de thèse.

Source : Enquêtes génération du Céreq, 2001, 2004 et 2007.

\subsection{Quand les conditions de réalisation de la thèse déterminent l'accès à l'emploi académique}

Tout d'abord, soutenir sa thèse en cinq ans ou plus a un effet fortement significatif et négatif, à la fois sur la probabilité d'accéder à l'emploi académique et d'être mobile. Autrement dit, plus un docteur met du temps à soutenir sa thèse, moins il a de chance d'être en poste trois ans plus tard dans l'ESR. Une thèse longue envoie donc un mauvais signal pour le recrutement académique.

Cet effet négatif est récent puisqu'il n'était pas significatif pour les générations 2001 et 2004, ce qui peut traduire un durcissement des critères dans l'accès aux postes académiques : réaliser une thèse longue constitue un handicap de plus en plus important pour accéder à l'ESR. Quant à l'effet négatif de la durée de thèse sur la mobilité, il confirme l'idée d'un ancrage spatial et relationnel plus fort lorsque le docteur avance en âge ${ }^{10}$.

Concernant les financements, il semblerait qu'un financement accompagné d'une activité d'enseignement représente la meilleure configuration pour accéder à l'ESR. Ne pas avoir enseigné, avoir réalisé une thèse avec un financement CIFRE ou sans financement sont autant d'éléments qui réduisent significativement les perspectives de carrière académique.

Dans le même temps, du côté des financements, on observe des effets globalement stables entre les générations, avec tout de même un rôle décroissant de l'enseignement comme critère d'accès à l'ESR. En effet, un docteur qui a réalisé une thèse financée, sans

10. En effet, l'introduction de la variable âge dans le modèle capture l'effet de la durée de thèse, qui n'est plus significatif, ni sur l'accès à l'ESR, ni sur la mobilité. La variable âge n'a pas été testée dans le présent modèle car elle est très corrélée (au seuil de $1 \%$ ) avec la variable de durée de la thèse. Pour corriger l'effet de durée de la thèse, il aurait fallu calculer l'âge à l'entrée en thèse, mais les données ne nous le permettent pas. 
enseignement, relativement à un docteur qui a enseigné, a une probabilité significativement plus faible d'obtenir un poste académique pour les générations 2001 et 2004, et l'effet disparaît pour la génération 2007. On peut penser que les critères de recrutement sont de plus en plus orientés vers le profil scientifique des candidats, ce que la variable relative aux publications semble confirmer.

En effet, le nombre de publications représente une variable déterminante de l'accès à l'ESR. À l'ESR, $30 \%$ des docteurs non publiants sont en poste trois ans après leur thèse, contre $45 \%$ des docteurs qui comptabilisent une ou deux publications et $50 \%$ de ceux ayant trois publications ou plus. L'activité de publication constitue donc un critère essentiel dans le recrutement des universitaires. Notons que la proportion de nonpubliants ayant un projet hors ESR est plus forte que la moyenne des docteurs, ce qui peut indiquer une forme d'anticipation dans la mise en œuvre du projet (Mangematin, op. cit.). Pour les générations 2001 et 2004, avoir publié, indépendamment du nombre de publications, augmente la probabilité d'avoir un poste dans l'ESR. Pour la génération 2007, avoir publié un ou deux articles n'a pas d'effet sur cette probabilité : c'est à partir de trois publications que le docteur a un accès facilité à l'ESR. Ce résultat indique que l'activité de publication doit être de plus en plus importante pour constituer un avantage lors des recrutements. Cette évolution peut être interprétée comme une réponse directe au caractère de plus en plus concurrentiel du marché académique lié à la baisse du nombre de postes offerts.

Le post-doctorat a un effet positif à la fois sur l'accès à l'ESR et sur la mobilité. Côté professionnel, notons que nous observons l'effet à trois ans après la fin de la thèse : le post-doctorat peut être considéré comme une étape nécessaire du parcours et constitue donc un avantage relatif par rapport à ceux qui n'en ont pas fait, mais retarde la stabilisation. L'effet du post-doctorat sur l'accès est observé pour les trois générations. Pour la mobilité, on peut légitimement penser que, dans une logique cumulative, l'expérience de la mobilité favorise la mobilité (Kaufmann, 2005). La réalisation d'un post-doctorat dans un autre laboratoire peut d'ailleurs conduire à un éventuel recrutement.

Le modèle met en évidence l'existence d'un effet discipline sur les carrières, les docteurs en DEG étant ceux qui accèdent le plus aux postes dans l'ESR relativement à leurs collègues de LSH et de sciences. Cela confirme les tendances observées par Béret et $a l$. (op. cit.) selon lesquelles les docteurs en DEG font de plus en plus carrière dans l'ESR, alors que la part des docteurs en LSH accédant aux postes d'enseignants-chercheurs a sensiblement baissé. L'analyse conjointe des générations 2001, 2004 et 2007 dans le présent article montre que la progression de la part des carrières académiques pour les docteurs en DEG se poursuit (+ 6 points) et qu'il y a eu une stabilisation côté LSH. Le modèle indique que les docteurs en sciences accèdent relativement moins aux postes académiques que les docteurs en DEG à partir de la génération 2004. 
Enfin, il apparaît que les projets professionnels des docteurs en fin de thèse ${ }^{11}$ sont corrélés avec leur situation trois ans plus tard. $86 \%$ (resp. 89\%) des docteurs projetant de travailler dans la recherche privée (resp. hors recherche) sont en emploi hors ESR. Notons que la part d'emploi titulaire dans l'ESR est plus forte pour les docteurs dont le projet était l'ESR (60 \%) que pour les docteurs qui n'en avaient pas formulé le souhait (39\%). Le taux d'accès à l'ESR pour les docteurs projetant de travailler dans la recherche publique est seulement de $54 \%$, ce qui témoigne de la relative difficulté à trouver un poste académique.

\section{Conclusion}

Cet article visait à identifier simultanément ${ }^{12}$ les déterminants de l'accès des jeunes docteurs aux différents marchés du travail et de leur éventuelle mobilité géographique, et leur évolution. Rappelons deux limites de cette étude, qu’on pourra chercher à dépasser dans le cadre de recherches futures. D'abord, la mobilité géographique ne prend pas en compte les départs à l'étranger. Ensuite, la trajectoire professionnelle a été réduite à l'accès ou non aux carrières académiques, sans étudier la stabilisation dans l'emploi.

Parmi les variables qui influencent à la fois les trajectoires professionnelles et géographiques des jeunes docteurs, on peut d'abord citer l'Ile-de-France comme région de soutenance, qui semble être la meilleure configuration pour accéder à l'ESR sans mobilité spatiale. La durée de thèse, ensuite, lorsqu'elle est supérieure ou égale à cinq années, impacte négativement la probabilité d'accéder à l'ESR et d'être mobile ; on pourrait parler de prime à la précocité dans le monde académique, l'avancée en âge, signe d'ancrage, rendant plus difficile la mobilité. Enfin, le post-doctorat constitue une première mobilité qui invite à rester dans le monde de l'ESR, plutôt dans le cadre de carrières non locales, c'est-à-dire sans retour dans la région d'origine.

Au final, on observe une forte segmentation des critères de recrutement selon que les docteurs s'insèrent ou non sur le marché de l'emploi académique. Nos résultats confirment l'hypothèse de Mangematin (op. cit.), selon laquelle il existence un relatif déterminisme une fois la thèse engagée, selon le type de financement notamment et le mode de valorisation choisi, les publications étant l'un des déterminants essentiels d'accès aux carrières académiques. L'analyse conjointe de trois générations a également permis de mettre en évidence que, pour accéder à l'ESR, l'importance accordée aux publications et à la durée de thèse s'est renforcée, au détriment de l'expérience d'enseignement. Ainsi, dans un contexte de plus en plus concurrentiel, l'accent serait donc davantage mis sur l'activité scientifique.

11. Le projet en sortie de thèse peut paraître endogène, il aurait été préférable de tester le projet professionnel en début de thèse, mais cette variable n'est pas disponible. Notons que le fait d'enlever cette variable du modèle ne modifie pas les effets des autres variables.

12. La justification économétrique de l'estimation d'un probit bivarié plutôt que deux probit simples séparés indique déjà une forme d'enchevêtrement des deux types de trajectoires, qui peuvent être affectées par des déterminants communs. 
Concernant les trajectoires géographiques, notons la relative inertie spatiale observée, puisque seulement un tiers des docteurs en activité travaillent trois ans après la fin de leur thèse dans une région différente de celle où ils l'ont soutenue. Ce score est largement inférieur à celui des diplômés de Master. En outre, une partie non négligeable des mobilités observées après la thèse correspond à des retours dans la région d'origine des docteurs. Si le groupe des variables sociodémographiques (genre, pays de naissance, père cadre, en couple) présente des effets plutôt instables selon les générations et les secteurs disciplinaires, les variables d'ordre géographique ont quant à elles des effets marqués sur la mobilité. Le fait de soutenir sa thèse dans sa région d'origine renforce significativement la probabilité d'y rester, pour les trois générations étudiées, que l'on soit docteur en SHS ou en sciences.

Si la question de la mobilité est majoritairement étudiée à l'échelle des individus, elle impacte également les systèmes d'emploi que les individus composent. Alors que l'Université est animée par le débat sur le recrutement local (Bouba-Olga et al., op. cit. ; Godechot et Louvet, $o p$. cit.), les résultats de notre recherche montrent que le taux de mobilité augmente significativement dans le temps et que les docteurs s'insérant hors ESR présentent un taux de mobilité inférieur de cinq points à celui de leurs collègues, quelle que soit la génération concernée. Dès lors, la faible mobilité n’est pas spécifique au monde de l'ESR. L'enchevêtrement avéré des trajectoires professionnelles et géographiques des jeunes docteurs, ainsi que l'intervention de déterminants personnels au moment où la carrière familiale se joue également (Bonnet et al., 2006), pourraient faire l'objet d'une analyse qualitative pour déconstruire plus finement les processus décisionnels des individus.

\section{Bibliographie}

Almeida P., Kogut B. (1999), "Localization of knowledge and the mobility of engineers in regional networks", Management Science, 45(7), pp. 905-917.

APEC (2014), «Le devenir professionnel des jeunes docteurs : quel cheminement, quelle insertion 5 ans après la thèse ? ", Les études de l'emploi cadre, 57.

Arntz M. (2010), "What attracts human capital? Understanding the skill composition of interregional job matches in Germany”, Regional Studies, 44(4), pp. 423-441.

Auriol L. (2010), "Careers of doctorate holders: employment and mobility patterns, OECD Science, Technology and Industry”, Working Papers, (2010)/04, OECD Publishing.

Autant-Bernard C., Massard N. (2001), "Externalités de connaissances et géographie de l'innovation : les enseignements des études empiriques ", Document de travail, CREUSET.

Baron M., Perret C. (2008), "Comportements migratoires des étudiants et des jeunes diplômés : ce que révèle le niveau régional », Géographie Economie Société, 10(2), 223-242.

Bauman Z. (2013), Liquid modernity, John Wiley and Sons. 
Becker G.S. (1962), "Investment in human capital: A theoretical analysis", Journal of Political Economy, 70(5), pp. 9-49.

Béduwé C. (1994), « Mobilité géographique des étudiants diplômés : probabilités individuelles et effets structurels ", Formation Emploi, 48, pp. 3-20.

Béret P., Giret J.-F., Recotillet I. (2004), « L'évolution des débouchés professionnels des docteurs : les enseignements de trois enquêtes du Céreq ", Éducation et Formations, 67, pp. 109-116.

Bernela B., Bouba-Olga O., Ferru M. (2017), "Spatial patterns of PhDs' internal migration in France, 1970-2000", Journal of Innovation Economics and Management, forthcoming.

Boltanski L., Chiapello E. (1999), Le nouvel esprit du capitalisme, Gallimard.

Bonnal L., Giret J.-F. (2009), «La stabilisation des jeunes docteurs sur le marché de l'emploi académique ", Revue d'Economie Politique, 119(3), pp. 373-400.

Bonnard C. (2012), "Jeunes diplômés en sciences : les déterminants de l'accès à la recherchedéveloppement privée ", Formation Emploi, 117, pp. 49-68.

Bonnet E., Collet B., Maurines B. (2006), "Carrière familiale et mobilité géographique professionnelle ", Cahiers du genre, 2, pp. 75-98.

Bouba-Olga O., Grossetti M., Lavigne A. (2008), « Le localisme dans le monde académique : une autre approche ", La Vie des Idées, 12 mai.

Boussard V. (2013), Injonction de mobilité et différenciation de carrière pour les cadres : le cas de la mobilité géographique, Rapport pour l'IRES et la CFE-CGC.

Dahl M.S., Sorenson O. (2010), "The social attachment to place”, Social Forces, 89(2), pp. 633-658.

David Q., Janiak A. Wasmer E. (2010), “Local social capital and geographical mobility”, Journal of Urban Economics, 68(2), pp. 191-204.

Debrand T., Taffin C. (2005), « Les facteurs structurels et conjoncturels de la mobilité résidentielle depuis 20 ans ", Economie et Statistique, 381, pp. 125-146.

Drapier C., Jayet H. (2002), « Les migrations des jeunes en phase d'insertion professionnelle en France. Une comparaison selon le niveau de qualification ", Revue d'Économie Régionale et Urbaine, 3, pp. 355-376.

Etzkowitz H., Leydesdorff L. (2000), “The dynamics of innovation: from National Systems and "Mode 2" to a Triple Helix of university-industry-government relations", Research Policy, 29(2), pp. 313-330.

Fox M. F., Stephan P. E. (2001), "Careers of young scientists: Preferences, prospects and realities by gender and field”, Social Studies of Science, 31(1), pp. 109-122. 
Génération 2001-2004 (2004), Centre Maurice Halbwachs (diffuseur), Céreq (producteur). Génération 2004-2007 (2007), Centre Maurice Halbwachs (diffuseur), Céreq (producteur). Génération 2007-2010 (2010), Centre Maurice Halbwachs (diffuseur), Céreq (producteur).

Gibbons M., Limoges C., Nowotny H., Schwartzman S., Scott P., Trow M. (1994), The New Production of Knowledge: the dynamics of science and research in contemporary societies. SAGE, London.

Giret J.-F. (2005), De la thèse a l'emploi : les débuts professionnels des jeunes titulaires d'un doctorat, Céreq-Bref, n²20.

Giret J.-F., Perret C., Recotillet I. (2007), « Le recrutement des jeunes docteurs dans le secteur privé », Revue d'Economie Industrielle, 119(3), pp. 85-102.

Godechot O., Louvet A. (2008), «Le localisme dans le monde académique : un essai d'évaluation ", La Vie des Idées, 22 avril.

Grossetti M. (1991), «Trajectoires d'ingénieurs et territoire. L'exemple des hautes technologies à Toulouse ", Sociétés contemporaines, 6, pp. 65-80.

Grossetti M., Eckert D., Gingras Y., Jégou L., Larivière V., Milard B. (2014), “Cities and the geographical deconcentration of scientific activity: A multilevel analysis of publications (1987-2007)", Urban Studies, 51(10), pp. 2219-2234.

Guellec D., Cervantes M. (2002), "International mobility of highly skilled workers: From statistical analysis to policy formulation", OECD - International mobility of the highly skilled, pp. 71-98.

Harfi M., Mathieu C. (2006), « Mobilité internationale et attractivité des étudiants et des chercheurs ", Horizons stratégiques, 1(1), pp. 28-42.

Herzog J.H.W., Schlottmann A.M., Boehm T.P. (1993), "Migration as spatial job-search: a survey of empirical findings", Regional Studies, 27(4), pp. 327-340.

Kaufmann V. (2005), " Mobilités et réversibilités : vers des sociétés plus fluides ? ", Cahiers internationaux de sociologie, 1, pp. 119-135.

Lemistre P., Magrini M.-B. (2010), « Mobilité géographique des jeunes : du système éducatif à l'emploi, une approche coûts/bénéfices des distances parcourues ", Formation Emploi, 110, pp. 63-78.

Lippmann S.A., McCall J.J. (1976), “The economics of job search: a survey”, Economic Inquiry, 14(2), pp. 155-367.

Lollivier S. (2001), « Endogénéité d'une variable explicative dichotomique dans le cadre d'un modèle probit bivarié : une application au lien entre fécondité et activité féminine ", Annales d'Economie et de Statistique, pp. 251-269. 
Mangematin V. (2000), "PhD job market: professional trajectories and incentives during the PhD", Research Policy, 29, pp. 741-756.

Marry C. (2008), « Le plafond de verre dans le monde académique : l'exemple de la biologie ", Idées économiques et sociales, 3, pp. 36-47.

MESR (2012), Campagne de recrutement et d'affectation des maîtres de conférences et des professeurs des universités, Rapport de la DGRH A1-1.

MESR (2013), Etude de la promotion 2012 des qualifiés aux fonctions de maître de conférences et de professeur des universités, Rapport de la DGRH A1-1.

Mincer J. (1978), "Family migration decisions", Journal of Political Economy, 86(5), pp. $749-773$.

Perret C. (2003), Mobilités géographiques sur le territoire national et insertion professionnelle des jeunes docteurs, Relief 2, Rapport du Céreq.

Pochic S. (2005), «Faire carrière : l'apport d'une approche en termes de genre », Formation Emploi, 91, pp. 75-93.

Recotillet I. (2007), "PhD graduates with post-doctoral qualification in the private sector: does it pay off?", Labour, 21(3), pp. 473-502.

Riedinger N., Zaiem M. (2011), "Y a-t-il un problème d'insertion des titulaires de doctorat dans les centres de R\&D des entreprises? ", Economie et Prévision, 1, pp. 177-184.

Roach M., Sauermann H. (2010), “A taste for science? PhD scientists' academic orientation and self-selection into research careers in industry", Research Policy, 39(3), pp. 422-434.

Robin S., Cahuzac E. (2003), "Knocking on academia's doors: an inquiry into the early careers of doctors in life sciences", Labour, 17(1), pp. 1-23.

Schwartz A. (1973), "Interpreting the effect of distance on migration", Journal of Political Economy, 81(5), pp. 1153-1169.

Shauman K.A., Xie Y. (1996), "Geographic mobility of scientists: sex differences and family constraints”, Demography, 33(4), pp. 455-468.

Sjaastad L.A. (1962), "The costs and returns of human migration", Journal of Political Economy, 70(4), pp. 80-93.

Stephan P.E. (1996), "The economics of science", Journal of Economic Literature, 34(3), 1199-1262.

Vincent S., Viry G., Kaufmann V. (2010), "Carrières académiques : comment concilier mobilités spatiales et vie de famille? ", Revue Synergies, Pays Riverains de la Baltique, 7, pp. 77-9. 